\title{
Proper motions and velocity asymmetries in the RW Aur jet ${ }^{\star}$
}

\author{
L. López-Martín ${ }^{1,2}$, S. Cabrit ${ }^{2}$, and C. Dougados ${ }^{3}$ \\ 1 Instituto de Astrofísica de Canarias, c/ Vía Láctea s/n 38200, La Laguna, Tenerife, Spain \\ 2 LERMA, Observatoire de Paris, UMR 8112 du CNRS, 61 Av. de l'Observatoire, 75014 Paris, France \\ 3 Laboratoire d'Astrophysique, Observatoire de Grenoble, UMR 5571, BP 53, 38041 Grenoble Cedex 9, France
}

Received 4 April 2003 / Accepted 7 May 2003

\begin{abstract}
We present adaptive optics spectro-imaging observations of the RW Aur jet in optical forbidden lines, at an angular resolution of $0.4^{\prime \prime}$. Comparison with HST data taken 2 years later shows that proper motions in the blueshifted and redshifted lobes are in the same ratio as their radial velocities, a direct proof that the velocity asymmetry in this jet is real and not an emissivity effect. The inferred jet inclination to the line of sight is $i=46 \pm 3^{\circ}$. The inner knot spacing appears best explained by time variability with at least two modes: one irregular and asymmetric (possibly random) on timescales of $\leq 3-10 \mathrm{yr}$, and another more regular with $\simeq 20 \mathrm{yr}$ period. We also report indirect evidence for correlated velocity and excitation gradients in the redshifted lobe, possibly related to the blue/red velocity and brightness asymmetry in this system.
\end{abstract}

Key words. techniques: high angular resolution - stars: pre-main sequence - stars: individual: RW Aur ISM: jets and outflows - ISM: individual objects: HH229

\section{Introduction}

Jets from young stars are believed to be powered by accretion and accelerated and collimated by magnetic forces (see e.g. Königl \& Pudritz 2000 for a review). However, a clear identification of the magnetic launch configuration is still needed to fully understand the role of jets in the physics of accreting protoplanetary disks. The optically revealed innermost jet regions of T Tauri stars (TTS) hold essential clues to this question, and are the subject of growing observational efforts at sub-arcsecond resolution (e.g. Lavalley-Fouquet et al. 2000; Dougados et al. 2000 (hereafter Paper I); Bacciotti et al. 2000). HH 229, the bipolar jet from the actively accreting star RW Aur A, is a particularly interesting target. It was first identified in long-slit spectroscopy by Hirth et al. (1994), who noted an asymmetry in radial velocity between the blueshifted and redshifted lobes of a factor $\simeq 1.8$. Further study with HST/STIS (Woitas et al. 2002, hereafter Paper II) traces this asymmetry down to $0.1^{\prime \prime} \approx 14 \mathrm{AU}$ from the source, suggesting that it might be an intrinsic property of the jet driving engine. In addition, the RW Aur jet contains many emission knots, traced as far as 50"-100" (Mundt \& Eislöffel 1998) down to only $0.1^{\prime \prime}-$ $10^{\prime \prime}$ from the star (Papers I and II), which may be used to test models of knot formation in stellar jets.

Send offprint requests to: L. López-Martín,

e-mail: luislm@ll.iac.es

* Based on observations collected at the Canada-France-Hawaii Telescope (CFHT), operated by the National Research Council of Canada, the Centre National de la Recherche Scientifique, and University of Hawaii.
In this Letter, we present a ground-based spectro-imaging study of the RW Aur jet that brings several new results, including improved knot proper motions, jet inclination, and knot dynamical times, as well as indirect evidence for internal velocity and excitation gradients. Our observations are summarized in Sect. 2 and our results are discussed in Sect. 3.

\section{Observations}

Observations were carried out at the CFHT on December 18-20, 1998 with the integral field spectrograph OASIS. We used a spatial sampling of $0.16^{\prime \prime} /$ lens and achieved an angular resolution of $0.4^{\prime \prime}$ FWHM after adaptative optics correction. Two spectral ranges were observed (6213-6534 $\AA$ and 6496-6824 $\AA$ ) with a resolving power $\approx 90 \mathrm{~km} \mathrm{~s}^{-1}$. A second dataset with $0.11^{\prime \prime} /$ lens was obtained over a smaller field of view. Following standard OASIS reduction (cf. Lavalley-Fouquet et al. 2000), a specific procedure was applied to subtract at each position the strong continuum+emission spectrum of RW Aur A, scaled by the PSF, and to construct datacubes of residual forbidden line profiles. More details are given in Dougados et al. (2003, in preparation). Comparison between the two lens samplings shows that continuum-subtracted emission maps at $0.16^{\prime \prime} /$ lens are reliable beyond $0.35^{\prime \prime}$ from the star, while the $0.11^{\prime \prime} /$ lens data are more reliable at $d<0.35^{\prime \prime}$. Radial velocities are computed in the stellar reference frame (heliocentric velocity $+16 \mathrm{~km} \mathrm{~s}^{-1}$; Petrov et al. 2001). We adopt hereafter the notation $[\mathrm{O} \mathrm{I}] \equiv[\mathrm{O} \mathrm{I}] \lambda 6300$, $[\mathrm{S} \mathrm{II}] \equiv[\mathrm{S} \mathrm{II}] \lambda 6731$, and $[\mathrm{N} \mathrm{II}] \equiv[\mathrm{N} \mathrm{II}] \lambda 6583$. 


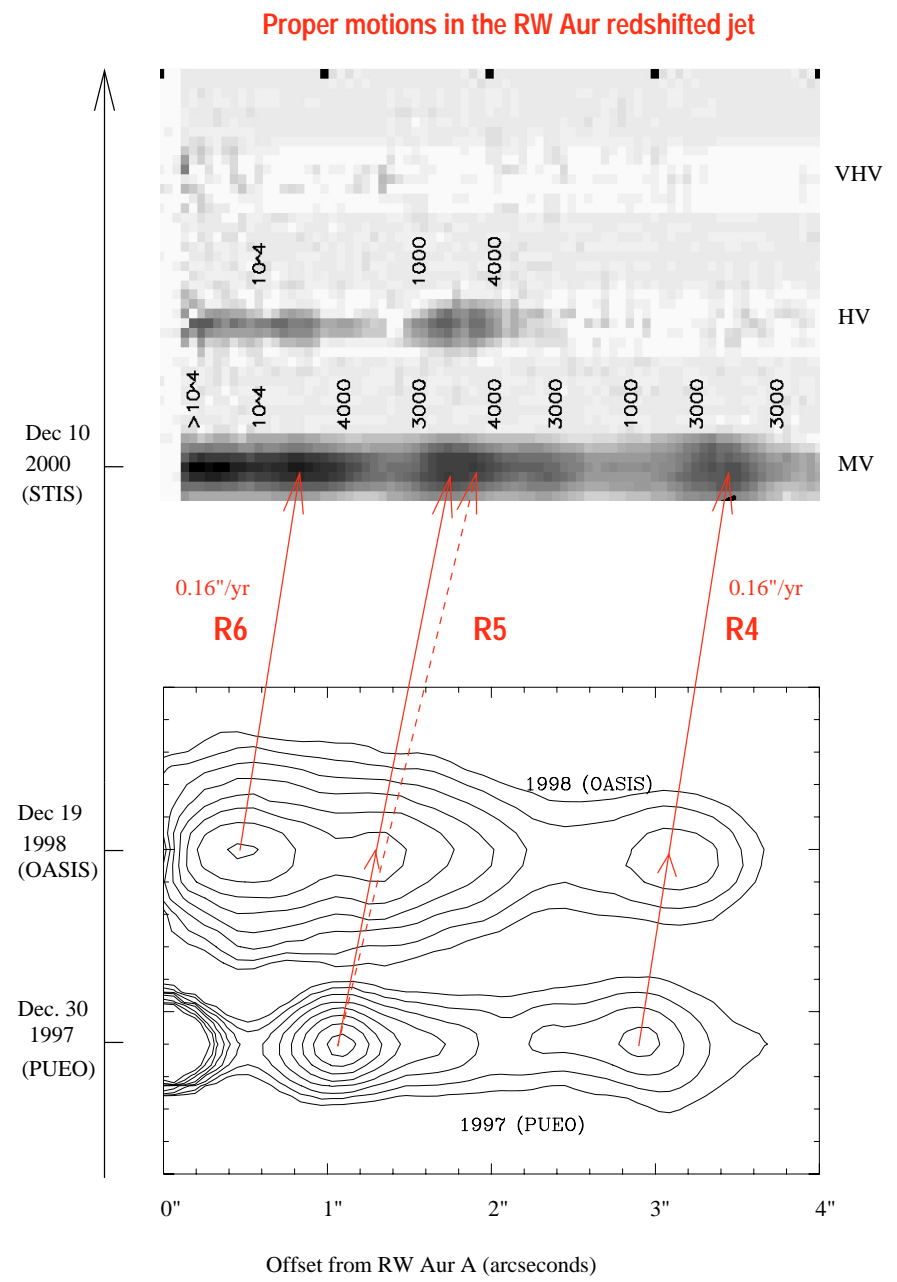

Fig. 1. Illustration of knot proper motions in the inner redshifted jet of RW Aur. From top to bottom: [S II] channel maps obtained in Dec. 2000 with STIS/HST (Paper II); integrated [S II] image obtained in Dec. 1998 with OASIS at 0.16"/lens (this paper); deconvolved [O I] image obtained in Dec. 1997 with PUEO at CFHT (Paper I).

\section{Results}

\subsection{Proper motions of redshifted knots}

Figure 1 compares our 1998 [S II] image of the redshifted jet with images obtained in Dec. 2000 (Paper II) and in Dec. 1997 (Paper I $)^{1}$. A smooth systematic shift is seen between the 3 epochs, indicative of proper motions. Table 1 lists the knot positions and the resulting proper motions in arcsec per year. As the OASIS spatial resolution is not sufficient to detect possible offsets between different lines, we adopt for the nominal 1998 position of each knot in Table 1 an average of independent estimates using the strongest two lines ([O I] and [S II]) and the two lens samplings $\left(0.11^{\prime \prime}\right.$ and $\left.0.16^{\prime \prime}\right)$. Knots are numbered from the outside in, starting at the most distant one detected by Dougados et al. (Paper I). For the outermost knot seen with OASIS (knot R4), the 3 epochs are consistent with a constant proper motion of $0.16^{\prime \prime} \mathrm{yr}^{-1}$. The proper motion for

\footnotetext{
${ }^{1}$ In order to replicate the correct separation between RW Aur A and B, the Dec. 1997 image published in Paper I had to be corrected for a small 5\% underestimate in the FOCAM pixel size.
}

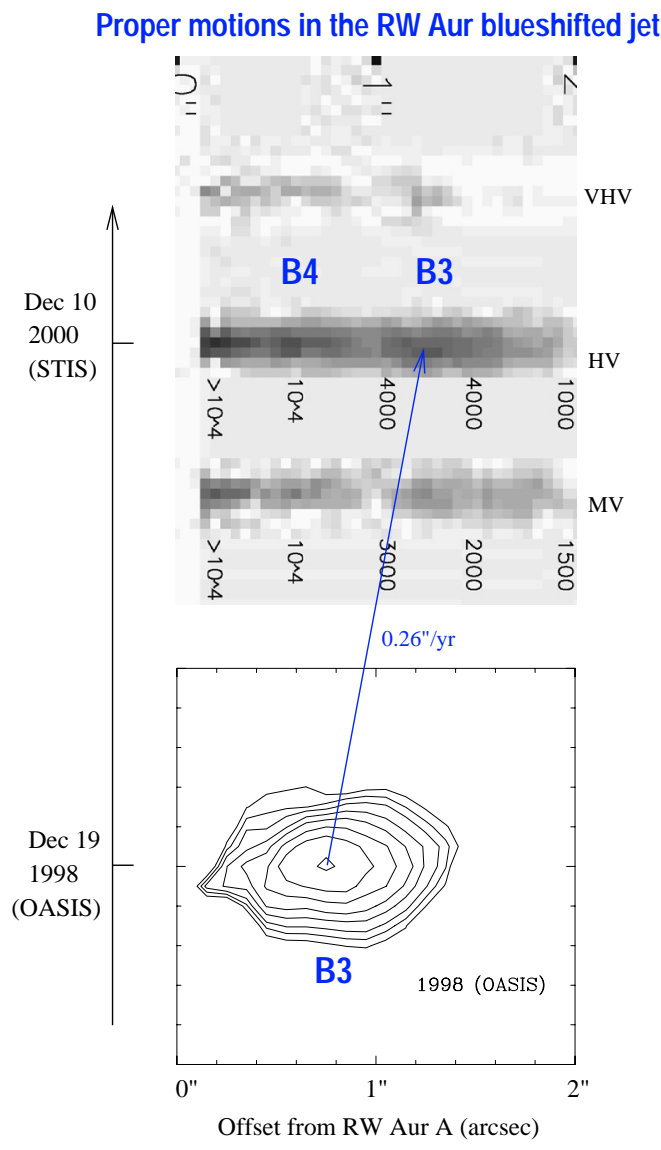

Fig. 2. Comparison of [S II] images of the RW Aur blueshifted jet obtained with OASIS in Dec. 1998 (this paper) and with STIS/HST in Dec. 2000 (Paper II), illustrating the proper motion.

the following knot (R5) is more uncertain, as it appears double in the 2000 STIS image. The range of possible values is $0.17^{\prime \prime}-0.27^{\prime \prime} \mathrm{yr}^{-1}$. For knot R6, we find again $0.16^{\prime \prime} \mathrm{yr}^{-1}$, based on the 1998 and 2000 epochs only. We note that an innermost emission peak is detected in the $0.11^{\prime \prime} /$ lens dataset at $\simeq 0.28^{\prime \prime}$. It may correspond to the peak at $0.25^{\prime \prime}$ in the STIS image, and would then be stationary.

\subsection{Proper motions of blueshifted knots}

Figure 2 compares [S II] images of the inner blueshifted jet in 1998 (this paper) and 2000 (Paper II). We lack a 1997 epoch, as deconvolution residuals in the PUEO image dominate over the faint blue jet emission out to $\simeq 2$ " (Paper I). We find a proper motion of $0.26^{\prime \prime} \mathrm{yr}^{-1}$ for the main knot (B3). A new knot (B4) has appeared at $0.6^{\prime \prime}$ in the 2000 STIS image. With a similar proper motion to B3, it would have been within $0.05^{\prime \prime}$ of the star at the date of the OASIS observations and thus unresolvable. Finally, our $0.11^{\prime \prime} /$ lens dataset shows an innermost peak at $\simeq 0.19^{\prime \prime}$, which may correspond to the $0.2^{\prime \prime}$ peak seen in the STIS image and would then be stationary. 
Table 1. Properties of knots in the RW Aur jet. $d_{97}, d_{98}$, and $d_{00}$ are the projected knot offsets in Dec. 1997, 1998, and 2000 (Paper I; this paper; Paper II), with respective uncertainties $\pm 0.01^{\prime \prime}, \pm 0.02^{\prime \prime}$, and $\pm 0.05^{\prime \prime}$, unless when quoted. $V_{\text {rad }}$ is the knot [S II] centroid velocity from this paper (roman font) and from Paper II (italics); $\mu$ the proper motion (typical uncertainty $\pm 0.027^{\prime \prime} \mathrm{yr}^{-1}$; values between brackets are assumed); $i$ the angle to the line of sight (error bars include uncertainties in both $\mu$ and $V_{\text {rad }}$ ); $t_{98}-t_{\mathrm{ej}}$ the time between knot "ejection" and Dec. $20,1998$.

\begin{tabular}{|c|c|c|c|c|c|c|c|}
\hline Knot & $\begin{array}{l}d_{97} \\
\left({ }^{\prime \prime}\right)\end{array}$ & $\begin{array}{l}d_{98} \\
\left({ }^{\prime \prime}\right)\end{array}$ & $\begin{array}{l}d_{00} \\
\left({ }^{\prime \prime}\right)\end{array}$ & $\begin{array}{c}\left|V_{\text {rad }}\right| \\
\left(\mathrm{km} \mathrm{s}^{-1}\right)\end{array}$ & $\begin{array}{c}\mu \\
\left({ }^{\prime \prime} \mathrm{yr}^{-1}\right)\end{array}$ & $\begin{array}{c}i \\
\left(^{\circ}\right)\end{array}$ & $\begin{array}{c}t_{98}-t_{\mathrm{ej}} \\
(\mathrm{yr})\end{array}$ \\
\hline \multicolumn{8}{|c|}{ Red lobe } \\
\hline & & $\leq 0.28$ & 0.25 & $100-96$ & stationary? & & \\
\hline R6 & & 0.51 & 0.82 & $94-117$ & 0.16 & $44 \pm 8$ & 1.3 \\
\hline R5 & 1.07: & 1.32 & $1.72 / 1.91$ & $107-133$ & $0.24 \pm 0.05$ & $53 \pm 9$ & $8 / 5$ \\
\hline R4 & 2.92 & 3.12 & 3.45 & $97-100$ & 0.16 & $47 \pm 5$ & 18 \\
\hline R3 & $3.9-4.6$ & & & & {$[0.16]$} & & $24-29$ \\
\hline $\mathrm{R} 2$ & 7.9 & & & & {$[0.16]$} & & 49 \\
\hline $\mathrm{R} 1$ & 11.1 & & & & {$[0.16]$} & & 69 \\
\hline \multicolumn{8}{|c|}{ Blue lobe } \\
\hline & & $\leq 0.19$ & 0.2 & $175-168$ & stationary? & & \\
\hline B4 & & & 0.57 & 179 & [0.26] & & 0.2 \\
\hline B3 & & $0.73 \pm 0.05$ & 1.25 & $178-168$ & $0.26 \pm 0.035$ & $45 \pm 5$ & 3 \\
\hline B2 & 2.8 & & & & {$[0.26]$} & & 12 \\
\hline B1 & 7.1 & & & & {$[0.26]$} & & 28 \\
\hline
\end{tabular}

\subsection{Revised jet inclination}

Assuming that knot proper motions trace actual fluid motions and not wave pattern speeds, the angle of inclination $i$ of the jet with respect to the line of sight is given by $\tan (i)=$ $\left(\mu /^{\prime \prime} \mathrm{yr}^{-1}\right) \times\left(666.7 / V_{\mathrm{rad}}\right)$, where $V_{\text {rad }}$ is the velocity projected on the line of sight, and a distance of $140 \mathrm{pc}$ to Taurus-Auriga is assumed. We adopt as $V_{\text {rad }}$ for each knot the centroid of a gaussian fit to the [S II] line profile. Values from this paper and from Paper II (in italics) are given in Col. 5 of Table 1. Discrepancies in $V_{\text {rad }}$ between the two datasets are discussed in more detail in Sect. 3.5. The resulting values of $i$ are given in Col. 7, with an uncertainty interval that includes both the uncertainty in $V_{\text {rad }}$ and that in $\mu$ (see notes to Table 1 ). Inclination values are remarkably consistent between knots, within the estimated errors. In particular, we derive consistent inclinations for the blue and red lobes, i.e. the proper motion in the blue jet is higher than in the red jet by the same factor $(\simeq 1.6)$ as their average centroid radial velocities. This fact argues strongly for a real velocity difference between the blueshifted and redshifted jets, rather than an apparent difference due solely to slow bowshock wings dominating the emission around the redshifted jet (proper motions should then be similar in the two lobes despite differing radial velocities). A weighted average over both lobes yields $^{2} i=46^{\circ} \pm 3^{\circ}$.

\subsection{Knot spacing, dynamical times, and origin}

The RW Aur observations allow to measure knot spacings and dynamical timescales very close to the source and thus bring

${ }^{2}$ The larger inclination value $i=53^{\circ}$ found by Woitas et al. (Paper II) for knot R4 stems from a proper motion estimate $20 \%$ too high, inferred from the published PUEO image of Paper I before correction for pixel scale (see footnote 1 ). interesting constraints on the origin of knots in stellar jets. To increase the distance and time span in our analysis, we include in Table 1 more distant knots imaged with PUEO in 1997, which lie beyond the OASIS field of view and were not considered by Woitas et al. (Paper II).

Table 1 shows that the knot spacing first increases with distance from the star, from $0.7^{\prime \prime}-0.8^{\prime \prime}$ at $d \simeq 1.5^{\prime \prime}$ (knots R5-R6, B3-B4) to $1.8^{\prime \prime}-2.4^{\prime \prime}$ at $d \simeq 3^{\prime \prime}$ (knots R4-R5, B2-B3), and then levels off at $\Delta x \simeq 3^{\prime \prime}-4^{\prime \prime}$ for $d \geq 4^{\prime \prime}$. Adopting as the jet characteristic radius $r_{\mathrm{j}}$ half the FWHM measured at the same distances in Paper II, the inner knot spacing is then $\Delta x \simeq 8-$ $10 r_{\mathrm{j}}$. For comparison, the axisymmetric pinch instabilities in MHD jets simulated by Lery et al. (2000) produce jet knot spacings of $\Delta x / r_{\mathrm{j}} \leq 3$, while Kelvin-Helmholtz instabilities predict $\Delta x / r_{\mathrm{j}} \simeq V_{\mathrm{j}} / c_{\mathrm{s}}>10$. An even more important observational constraint is that the first moving knot appears at a distance $d_{\min }<\Delta x$, while the growth scale of an instability is expected to be several times its wavelength. Hence, neither kind of instability seems adequate to explain the innermost knot spacings, although a range of MHD jet configurations should be explored before they are fully ruled out.

Another possible origin for the knots, which can easily yield $d_{\text {min }}<\Delta x$, is time variability in the jet ejection speed (cf. Eq. (3) in Raga et al. 1990). The last column of Table 1 gives the dynamical ages of each knot assuming constant proper motion (with $t=0$ arbitrarily set at our OASIS observation date of Dec. 19, 1998). For knots lacking proper motion measurements, we adopt the typical $\mu$ in the same lobe (in brackets in Col. 6 of Table 1). We find that the timescale between knots initially increases with distance, from 3 to $10 \mathrm{yr}$, and then appears to level off at $\simeq 20 \mathrm{yr}$ beyond $4^{\prime \prime}$. Furthermore, the inner knots do not appear to be "synchronized" between the two jet sides, while there is tentative evidence of synchronization when the $20 \mathrm{yr}$ period appears (knots R3 and B1). We conclude 


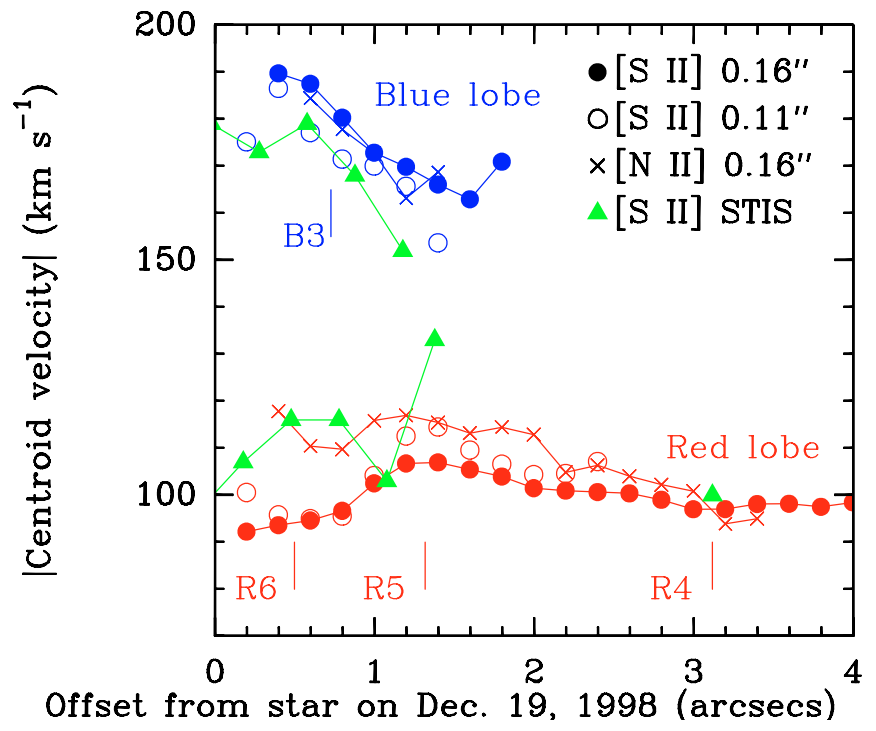

Fig. 3. Centroid radial velocity in [S II] (from gaussian fits) as a function of distance from the star. Filled and open circles refer to the $0.16^{\prime \prime} /$ pixel and $0.11^{\prime \prime} /$ pixel OASIS data, respectively, integrated over a $1^{\prime \prime}$ wide slit. For comparison, crosses show centroids in [N II] $\left(0.16^{\prime \prime} /\right.$ lens), and triangles show [S II] centroids in a $0.1^{\prime \prime}$ slit with HST/STIS (Woitas et al. 2002), shifted to correct for proper motion (by $-0.32^{\prime \prime}$ in the red lobe, $-0.52^{\prime \prime}$ in the blue; cf. Table 1).

that there would be two distinct processes of time variability in RW Aur: one that is irregular and asymmetric - possibly random (random time variability of the jet speed produces asymptotically an increase in knot spacing with distance; Raga 1992) and another more regular and symmetrical with a $20 \mathrm{yr}$ period - possibly related to a stellar magnetic cycle. We suggest that asynchronous variability might occur, e.g., through local magnetic reconnexion events that affect only one jet side.

The apparently stationary knots at $0.2^{\prime \prime}-0.3^{\prime \prime}=30-45 \mathrm{AU}$ from the star need to have a different origin, possibly recollimation shocks (e.g. Gomez de Castro \& Pudritz 1993) or temperature gradients in a progressively heated wind (e.g. Garcia et al. 2001), but predicted distances are presently too modeldependent to yield useful constraints.

\subsection{Velocity gradients and velocity asymmetry}

Figure 3 plots the [S II] centroid radial velocity as a function of distance from the star for the OASIS and STIS datasets (the latter is shifted to correct for proper motions). In the blue jet, the various datasets agree to within $10 \mathrm{~km} \mathrm{~s}^{-1}$, comparable to expected instrumental and calibration errors. The situation is different in the redshifted jet: the $0.11^{\prime \prime} /$ lens and $0.16^{\prime \prime} /$ lens datasets still agree with each other within $\leq 10 \mathrm{~km} \mathrm{~s}^{-1}$, but they lie typically $20 \mathrm{~km} \mathrm{~s}^{-1}$ below the STIS values out to the position of knot R5. Further out, however, at the position of knot R4, all data converge to a very similar $V_{\text {rad }}$.

These discrepancies can only be understood if the faster gas seen on the redshifted jet axis by STIS ( $0.1^{\prime \prime}$ wide slit) suffers from beam-smearing in the OASIS data. In other words, sharp transverse velocity gradients must occur on scales $\leq 0.4^{\prime \prime}$ within the redshifted jet, out to distances $<3^{\prime \prime}$ from the $s \operatorname{sar}^{3}$. We note that $[\mathrm{N} \mathrm{II}]$ centroids from OASIS (shown as crosses in Fig. 3) agree better with the [S II] STIS centroids, despite an angular resolution identical to the [S II] OASIS data. This fact further indicates that the faster axial gas contributes a greater fraction of the total flux in [N II] than in [S II], i.e. that it has higher excitation. Interestingly, we find no evidence for such velocity and excitation gradients in the blueshifted jet. If confirmed, this difference might be related to the blue/red velocity asymmetry between the two jet lobes; e.g., bowshock wings or turbulent boundary-layers could be somehow enhanced in the slower lobe (thus also explaining its higher brightness). Further theoretical and observational work is needed to explore this possibility.

Acknowledgements. LLM is grateful to Observatoire de Paris and to Ministère de l'Éducation Nationale for a postdoctoral fellowship.

\section{References}

Bacciotti, F., Mundt, R., Ray, T. P., et al. 2000, ApJ, 537, 49

Dougados, C., Cabrit, S., Lavalley, C., \& Ménard, F. 2000, A\&A, 346, 260 (Paper I)

Garcia, P. J. V., Cabrit, S., Ferreira, J., \& Binette, L. 2001, A\&A, 377, 609

Gomez de Castro, A. I., \& Pudritz, R. 1993, ApJ, 409, 748

Königl, A., \& Pudritz, R. 2000, PPIV (U. of Arizona Press), ed. V. Mannings, A. P. Boss, \& S. S. Russell, 759

Hirth, G. A., Mundt, R., Solf, J., \& Ray, T. P. 1994, ApJ, 427, L99

Lavalley-Fouquet, C., Cabrit, S. \& Dougados, C. 2000, A\&A, 356, L41

Lery, T., \& Frank, A. 2000, ApJ, 533, 897

Mundt, R., \& Eislöffel, J. 1998, AJ, 116, 860

Petrov, P. P., Gahm, G. F., Gameiro, et al. 2001, A\&A, 369, 993

Raga, A. C. 1992, MNRAS, 258, 301

Raga, A. C., Binette, L., Cantó, J., \& Calvet, N. 1990, ApJ, 364, 601

Woitas, J., Ray, T. P., Bacciotti, F., Davis, C., \& Eisloeffel, J. 2002, ApJ, 580, 336 (Paper II)

\footnotetext{
${ }^{3}$ Such gradients seem confirmed by preliminary analysis of transverse STIS slits (Woitas et al., work in progress).
} 